\title{
Correspondence
}

\section{Capacity assessments - diagnosis is misleading}

Ayre et al ${ }^{1}$ attempt to support the usefulness of the assessment of capacity in borderline personality disorder by validating it as a diagnostic construct. This is a flawed approach for several reasons.

- People with a diagnosis can move in and out of states of competence as much as those of us without a diagnosis; what would the Mental Capacity Act (MCA) have to say about a newly bereaved mother with suicidal thoughts (other than require us to invent a disorder of mind for her)?

- Acute presentations frequently preclude the ability to make a diagnosis; states of distress, psychoactive substances and lack of information all cloud our ability to make clear assessments; assessors vary in experience and competence and judgements about diagnosis vary among even those with comparable experience and skill.

- Because the MCA and popular culture privilege the place of diagnosis in determinations of responsibility, clinicians are led to first make a diagnosis and then, secondarily, make a determination of capacity - this results in predetermining capacity judgements based on diagnosis.

- Comorbidity is a frequent finding in personality disorder; however, the presence of Axis I disorder has the effect of 'trumping' the Axis II in the minds of professionals, the public and - crucially - patients, colouring patients' expectations of their ability to assume personal responsibility.

- Finally, there is an issue of tautology; capacity could not be impaired without some impairment of the individual's cognitive, perceptual or emotional state - the very abnormalities which indicate disorder of mind; the absence of capacity is thus sufficient to denote a disorder of mind and the requirement to name this directs clinicians to assign a diagnosis and attribute the incapacity to the diagnosis, rather than the aspect of function which impairs capacity.

We should be arguing to dissociate mental capacity from disorder of mind and, instead, deepen our thinking about the application of capacity judgements in clinical situations.

Graham M. Behr, Consultant Psychiatrist, Central and North West London NHS Foundation Trust, London, UK; email: graham.behr@nhs.net

1 Ayre K, Owen GS, Moran P. Mental capacity and borderline personality disorder. BJPsych Bull 2017; 41: 33-6.

doi: $10.1192 / \mathrm{pb} .41 .3 .181$

\section{Post-flight confusion}

Thomas McCabe describes a case of delirium apparently precipitated by flying. This is not unusual. Most liaison psychiatrists, especially old age liaison psychiatrists, will have seen several such cases and many of them after shorter flights. I reviewed the evidence for this in 2002 and 2012 and presented some ideas about how to proceed. ${ }^{2,3}$

Planes ascend to and descend from an altitude of between 30 and 40000 feet above sea level in 20 minutes; in-flight cabin environments are artificially controlled, except for ambient radiation which is higher at these altitudes. The controlled cabin pressures are equivalent to those between
6000 and 8000 feet above sea level. At these altitudes, there is a reduction of between 20 and $26 \%$ of available oxygen, resulting in haemoxyhaemoglobin saturations between 83 and $85 \%$ of normal. In fit people this can cause tachycardia, tachypnoea, headaches, dizziness, impaired coordination, fatigue and confusion. Reduced air pressure can also cause peripheral oedema and expansion of any air-filled spaces such as the bowel, sinuses and middle ear (Boyle's law).

Air humidity is reduced at altitude. The 'comfort zone' for humidity is between 50 and $65 \%$. A centrally heated room has about 25\% humidity and air cabin humidity is between 1 and $20 \%$. This can cause dehydration, hypovolaemia and reduced peripheral circulation.

Most research on passenger health has been done on fit young airmen, but the effect on the physiology of older or unwell people, or those with evidence of cerebrovascular disease, is unclear.

The Warsaw Convention (1929) states that airlines are not responsible for their customers' health; the fact that passengers are responsible for their own health may limit the industry's motivation to explore this further. The British Medical Association have published a review of the impact of flying on passengers' physical health. ${ }^{4}$

For some years I have advised my patients with dementia - especially those with significant vascular aetiology - to avoid flying if possible. If flying is essential, I advise that they sit near the front of the plane (where there is higher oxygen saturation), drink plenty of non-alcoholic fluids and move as much as possible during the flight.

I agree with many of Dr McCabe's recommendations. The most important next step is research into the prevalence of post-flight confusion, its prognosis and risk factors. This would not be difficult to do, although it may be controversial. For instance, people over the age of 65 embarking on flights of more than 4 hours could be asked to complete cognitive tests before departure, within a week of return and a few months after return. Any signs of confusion would indicate further investigations. It may be that those who experience sundowning or psychotic symptoms are more at risk, but this needs researching. Only by identifying the extent and associated risk factors of post-flight confusion can good advice be given to patients, travellers and the travel industry. More knowledge would not only give clues as to aetiology but also inform risk reduction.

Gianetta Rands, Consultant Psychiatrist, Specialist in Dementias and Mental Capacity, Re-cognition Health, London, UK; email: grands@ doctors.org.uk

1 McCabe T. 'Doc, can I fly to Australia?' A case report and review of delirium following long-haul flight. BJPsych Bull 2017; 41: 30-2.

2 Rands G. Fear of flying, reviewed. An example of evidence-based old age psychiatry. Psychiatrist 2002; 26: 188-90.

3 Rands G. Have we gone too far in translating ideas from aviation to patient safety? Letter. BMJ 2011; 342: c7309.

4 British Medical Association Board of Science and Education. The impact of flying on passenger health: a guide for healthcare professionals. BMA, 2004.

doi: 10.1192/pb.41.3.181a 